\title{
Reflexiones sobre la enseñanza de la gestión urbana: un ejercicio necesario para construir la ciudad
}

\author{
Reflexões sobre o ensino da gestão urbana: um exercício necessário para \\ construir a cidade
}
Reflections on the teaching urban management: a necessary exercise to build the city

Claudia Inés Carreño ${ }^{[0]}$, Armando Durán Durán ${ }^{[b]}$

[a] Universidad de los Andes, Bogotá, Colombia

[b] Universidad del Rosario Facultades de Ciencia Política y Gobierno y de Relaciones Internacionales, Programa de Gestión y Desarrollo Urbanos de la, Bogotá, Colombia

\section{Resumen}

El presente artículo responde al interés por construir un primer acercamiento a la cuestión sobre la enseñanza de la gestión urbana en Suramérica. Metodológicamente la pesquisa privilegió una perspectiva cualitativa, a partir de revisión documental y entrevistas en profundidad. Los principales hallazgos del estudio evidencian una marcada preocupación en las investigaciones que podrían generar pistas relativas a cómo mejorar el proceso de enseñanza de la gestión urbana. En este sentido, emergen pautas sobre la gestión urbana que reivindican la capacidad de creación de los sujetos sociales, esto es, de la agencia cotidiana. Otras perspectivas la ligan con estatutos más epistemológicos como su carácter inter y transdisciplinario. Estas pautas que se han erigido para significar la gestión de las ciudades en las últimas décadas, sin duda, coadyuvan en los procesos de enseñanza de la gestión urbana y, al mismo tiempo, imponen un desafío central: el de interrelacionar un saber sabio con un saber enseñar.

Palabras-clave: Gestión urbana. Enseñanza. Didáctica.

Resumo

Este artigo busca realizar uma aproximação inicial sobre a questão do ensino da gestão urbana na América do Sul. Metodologicamente, a pesquisa privilegiou uma perspectiva qualitativa, a partir da revisão de literatura e realização de entrevistas. As principais conclusões do estudo mostram uma preocupação acentuada em pesquisa para gerar pistas sobre como melhorar o ensino da gestão urbana. Nesse sentido, emergem pautas sobre a gestão urbana que reivindicam a capacidade de criação dos sujeitos sociais, isto é, da agência de todos os dias. Outras perspectivas a conectam com estatutos mais epistemológicos, como seu caráter inter

CIC é Comunicadora social-periodista, magíster en Planificación y Administración del Desarrollo Regional, doctora en Ciencias de la Educación, e-mail: ci.carreno49@uniandes.edu.co; claudiaines_carreno@hotmail.com

ADD é Sociólogo, magíster en Planificación y Administración del Desarrollo, doctor (c) en Sociología, e-mail: armando.duran@urosario.edu.co; barichara72@hotmail.com 
e transdisciplinar. Essas diretrizes, que foram desenvolvidas para traduzir a gestão das cidades nas últimas décadas, sem dúvida contribuem para o ensino da gestão urbana e ao mesmo tempo impõem um desafio central: encontrar um adequado inter-relacionamento entre um "saber de conhecimento" e um "saber pedagógico".

Palavras-chave: Gestão Urbana. Ensino. Didática.

\begin{abstract}
This article seeks to accomplish an initial approach on the issue of education of urban management in South America. Methodologically, the research favored a qualitative perspective, through literature review and interviews. The main findings of this study show a marked concern to generate clues about how to improve the teaching of urban management. In this sense, there is an emergence of guidelines on urban management claiming the ability to create social subjects, i.e., the everyday agency. Other prospects connect it with more epistemological statutes, such as its inter- and transdisciplinary nature. These guidelines, which were developed to translate the management of cities in recent decades, undoubtedly contribute to the teaching of urban management and, at the same time, pose a central challenge: To find a suitable inter-relationship between "knowledge expertise" and "pedagogical knowledge".
\end{abstract}

Keywords: Urban Management. Education. Teaching.

\section{Introducción}

Estudiar cómo se enseña en los posgrados, y específicamente cómo se enseña en los posgrados sobre gestión urbana en Suramérica, ha sido un tema poco indagado. En general, en materia de posgrados se ha investigado acerca de su oferta, calidad, reformas, acreditación y tendencias en lo educativo e impacto de la globalización (Fayad, 2010; Camou, 2009; Matallana \& Varela, 2006; Guadilla, 2003, 2014; Cataño, 2008; Krotsch, 2001; García de Fanelli et al., 2001; Marquis et al., 1998), mientras que en el cómo se enseña se ha privilegiado la reflexión desde las didácticas específicas de las matemáticas, la medicina y los idiomas.

De la consulta realizada en publicaciones científicas se identificaron siete estudios que aportan la reflexión sobre qué se entiende por gestión urbana (GEUR, 2012; Frey, 2012; Camargo, 2012; Ortega, 2008; Balbo, 2003; Jordan \& Simione, 1998); catorce pesquisas que ofrecen elementos para reflexionar sobre las prácticas de la enseñanza en el posgrado (Firmino, 2012; Domínguez, 2011; Stake, 2011; Cañedo et al., 2008; Kennedy, 2008; Gudmundsdóttir \& Shulman, 2005; Cols, 2004; Messina, 1999; Cashin, 1995; Hernández \& Sancho, 1993; Oliva \& Henson, 1989; Candau, 1987;
Fenstermacher, 1986); y cuatro indagaciones que reflexionan sobre la enseñanza de la gestión urbana (Frey, 2012; Carreño, 2012; Inzulza, 2011; Moya González, 1997).

Lo que se pretende con la revisión de dichas publicaciones y, además, con las consultas hechas a expertos, es poner en diálogo las categorías gestión urbana y enseñanza y, primordialmente, desatar interrogantes que contribuyan a pensar articuladamente estos procesos, de manera que nos permita idear "mejores" prácticas de enseñanza de la gestión urbana.

\section{Aportes al debate sobre el concepto de gestión urbana'}

La reflexión sobre la gestión urbana data de la década del sesenta del siglo anterior con la crisis del Estado de bienestar y de la planificación tradicional ${ }^{2}$.

\footnotetext{
1 Algunas evidencias que soportan este artículo se identificaron en el año 2013 cuando uno de los autores estaba vinculado a la Universidad Piloto de Colombia.

2 La planificación tradicional estaba basada esencialmente en predicciones, y muy armonizada con los principios de modernización de la vida social, racionalización de los procesos y secularización de las actividades humanas. Este paradigma
} 
Desde esta época, numerosos académicos han venido preguntándose por la cuestión de la gestión urbana: a quién involucra, qué significa, y qué representa su puesta en acción. Teniendo en cuenta esta preocupación, el presente artículo busca mapear algunos rasgos de este debate, tanto a través de lo consignado en diferentes publicaciones académicas como por medio de testimonios de expertos, los cuales fueron obtenidos en la investigación de campo que sustenta el presente artículo.

Quizá, dos hitos históricos hayan marcado las transformaciones contemporáneas por las que ha transitado la gestión urbana. El primero de ellos alude al papel central del Estado en la planificación de las naciones; se trata del enfoque de Necesidades Básicas (NB). Los gobiernos intentaron, de esta manera, diseñar una línea de acción gubernamental para asegurar a las poblaciones más vulnerables unas condiciones mínimas de vida. Para lograrlo se implementan y consolidan programas sociales destinados a atender las necesidades básicas de las personas: alimentación, salud, vivienda, empleo y educación. De igual forma, los Estados, siguiendo las orientaciones de los expertos, crean procedimientos y adoptan medidas para orientar la planificación de las ciudades. Con el advenimiento, a finales del siglo pasado, del Consenso de Washington ${ }^{3}$, se inicia una vuelta actualizada a los principios de la economía clásica, proferida en el siglo XVIII y XIX por Adam Smith y David Ricardo, principalmente. Esta perspectiva económica re-orientó la manera de concebir la gestión de los asentamientos y, con ello, surge el segundo hito histórico: el enfoque neoliberal. Desde una mirada que asignaba cierta responsabilidad a los Estados a la hora de satisfacer unos niveles mínimos de vida (Estado benefactor), se transita hacia un enfoque de corte neoliberal,

funcionó relativamente bien, dentro de lo esperado, durante las décadas del cincuenta y sesenta del siglo pasado. Sin embargo, desde principios de los años setenta los errores de predicción de la planificación han generado situaciones dramáticas (Fernández Güell, 1997 apud Parraguez et al., 2006), especialmente cuando se trata de tramitar situaciones locales y particulares desde unos planteamientos con pretensiones universalistas.

3 Conjunto de recomendaciones de política económica que fueron impulsadas por el gobierno norteamericano finalizando la década de los ochenta del siglo pasado. Tales propuestas destacan: disciplina fiscal; prioridades en el gasto público; ampliación de la base tributaria; liberalización del comercio y las finanzas; políticas de cambio competitivas; inversión extranjera directa; privatización; desregulación; y derechos de propiedad. en el que se privilegia una gestión descentralizada y compartimentalizada que necesita el repliegue del Estado (modernización del Estado) dejando en manos privadas (supuestamente más eficientes y eficaces) la planificación, diseño y gestión de aquellos procesos de los que anteriormente se responsabilizaba el gobierno.

Esta nueva "creencia" en los mercados, va constituyendo un saber dominante basado en la institución del mercado y en la confianza del sector privado como medios para auspiciar crecimiento y acumulación en los países. De igual forma, en la década del ochenta también se re-valoriza el modelo de administración ${ }^{4}$ de Estado que surgiera a principios del siglo $\mathrm{XX}$, y que retoma elementos de las organizaciones en términos "de la cultura del monopolio a la cultura de la competencia, de la cultura del ciudadano-servidor a la cultura del ciudadano-cliente [...] de la preocupación por la productividad a la calidad en la provisión de los servicios" (Santos, 2008, p. 82 apud Páez, 2013, s.p.). Pese a que esta perspectiva administrativa fue cuestionada durante las tres últimas décadas (Durán, 2012), el núcleo de esta visión permanece incólume, al entender la gestión como un proceso eficiente para administrar los "recursos humanos" necesarios para alcanzar una alta productividad por medio de los trabajadores, todo ello siguiendo protocolos muy precisos: división clara del trabajo; distribución de los deberes a través de una jerarquía administrativa supervisada; definición de un sistema de reglas y reglamentos; exclusión de lo personal en la actuación de los cargos; y comunicación vertical y formalizada entre puestos de trabajo. Es este modelo el que se convierte en referente de la gestión urbana, pues se supone que es símbolo y garantía de la eficiencia y la eficacia.

De acuerdo con Marcelo Balbo (2003) todo este nuevo modelo va generando transformaciones que impactan en las formas de concebir la gestión urbana, por lo que el estancamiento de la globalización

\footnotetext{
4 La escuela de la administración científica tuvo sus primeros desarrollos en los trabajos del ingeniero mecánico y economista estadounidense Frederick Winslow Taylor (1856-1915) y del ingeniero civil europeo Henry Fayol (1841-1925), principalmente. A partir de un estudio sistemático de la producción y del análisis de la operación de la manufactura y la fábrica, dichos autores definieron la administración como un proceso de orientación eficiente de los recursos (humanos, físicos, temporales y espaciales) necesarios para alcanzar una creciente productividad en el trabajo asalariado.
} 
económica y del mercado, y los procesos de urbanización acelerada hacen evidentes una serie de efectos y conflictos. El autor llama la atención especialmente sobre dos procesos que considera centrales. El primero se refiere a que con la reducción del Estado se alcanzó una mayor participación de los privados en el sector servicios, lo que llevó a una mayor eficiencia, calidad y oferta. Sin embargo también se generó un aumento en el precio de bienes y servicios, se dificultó el acceso y se incrementó la exclusión social. Como segundo tópico Balbo señala el paso de "ciudades de campesinos" a "ciudades de pobres" , fenómeno que ha traído una flexibilización del mercado, un aumento de la subocupación, y un trabajo precario para buena parte de los habitantes.

En toda esta transformación por la que atraviesan las sociedades, y particularmente las ciudades, algunos académicos se preguntan: ¿Qué es lo que en última instancia ha de perseguir la gestión urbana? Tratando de responder a dicha pregunta encontramos trabajos que intentan resignificar algunos de los conceptos tradicionales que se han usado para reflexionar sobre la gestión urbana. Un ejemplo de ello tiene que ver con la resignificación de la idea de administración, vinculándola con las categorías de la "función" y de lo "común". Como extensión del concepto de administración "se enriquece al incluir el 'espacio' (si puede llamarse así) que está entre el hecho físico, producto o servicio provisto en un extremo y en el otro, la institución de coordinación, provisión o administración" (Jordan \& Simione, 1998, p. 224). Para el GEUR (Guía Básica de la Gestión Urbana) sería "la suma de las muchas formas en que los individuos e instituciones públicas y privadas planifican y administran los asuntos comunes de la ciudad" (GEUR, 2012, p. 3).

Para Cristhian Ortega (2008) la gestión urbana se concibe como una interfaz entre la idea y la acción. Es un proceso eminentemente relacional que conecta la potencia de la idea con la fuerza de la realización, lo cual se hace evidente cuando piensa la gestión como "la evolución de la planificación como concepto y como logro de las demandas de los ciudadanos [es la puesta en marcha] de un conjunto

\footnotetext{
5 Pareciera que los relatos sobre la formación de la sociedad de mercado en la Europa Occidental de los siglos XI-XIX, contados por el historiador Robert Heilbroner, se actualizarán con los sucesos que caracterizan los procesos urbanos actuales en gran parte de este continente.
}

de acciones para el logro de un objetivo [...] es la fase entre el plan y el logro" (Ortega, 2008, p. 25). De igual manera, García Cruz \& Cárdenas (2004) agregan que la gestión urbana "agrupa estos procesos de acción encaminados al funcionamiento integral de la ciudad" (García Cruz \& Cárdenas, 2004, p. 104).

Dialogando con estas reflexiones e interpretaciones se arguye que la gestión urbana es mucho más que acción y emerge mucho antes que el plan (Carreño, 2013). La gestión estaría impregnada de las lógicas de la virtualidad que produce la imaginación y de la materialización que producen los actos humanos. De igual forma, y siguiendo a otros autores, es necesario involucrar nuevos debates que lleven a pensar la noción de la gestión desde su carácter de agencia. Desde la posibilidad de irrumpir lo instituido, esto es, el reconocimiento del sujeto gestor como creador, con la posibilidad de establecer nuevos consensos en medio de la diferencia, aunque sean temporales y en absoluto duraderos. En este sentido juega un papel central la "organización" de estas manifestaciones de cambio, de búsqueda de mejores condiciones de habitabilidad, que implica, entre otros aspectos, la movilización de "actores, intereses y voluntades para negociar en torno a unos objetivos de ciudad, con el fin de incidir en el mejoramiento de la calidad de vida" (Camargo, 2012, p. 5). Desde esta perspectiva de la gestión como posibilidad permanente de creación, de nuevas agencias de ciudad, es que emergen desafíos que relacionan, por ejemplo, los siguientes interrogantes: $¿$ Será posible idear formas de cooperación más horizontales donde los diferentes actores desplieguen valores relacionados con la justicia, la equidad y el bien común? ¿Es posible que los intereses individuales, personales, se supediten a intereses colectivos? Colocando la mirada en este horizonte se argumenta que la gestión urbana contemporánea ha de contemplar un gobierno que no sea exclusivo de las autoridades estatales, sino que más bien ataña a todos. En este sentido se propone a "la gobernanza, un tipo de gobierno más democrático; más participación de todos los grupos involucrados" [ex.: De acuerdo con Estudiante tres (Firmino, 2013)].

De igual forma surgen propuestas que acentúan la articulación de actores, instituciones y contextos de actuación social. Es el caso de la propuesta de Frey, quien concibe la gestión urbana como "todas las actividades desarrolladas por parte de los gobiernos locales, y realizarlas cada 
vez más en cooperación con actores privados, la sociedad civil y los ciudadanos en favor del desarrollo de las ciudades [su tarea fundamental] es la coordinación y articulación de las diferentes actividades, actores, sectores y saberes [...] son parte de la gestión urbana: la planificación urbana, el planeamiento sectorial, las políticas públicas como también la administración cotidiana de la Alcaldía". (Frey, 2012, p. 33). Sin embargo, queda aún la pregunta por el tipo de significado que se le otorga al planteamiento "en favor del desarrollo de las ciudades". Al final hemos de interrogarnos sobre aquello que para nosotros es desarrollo de lo urbano. Se sabe que llevamos casi siete décadas debatiendo sobre el desarrollo y durante todo este tiempo la noción se ha caracterizado de forma polisémica. El significado se lo disputan diferentes corrientes: liberales (el desarrollo como crecimiento económico al estilo de Walt Rostow), estructurales (quizá la principal teoría de esta vertiente es la cepalina denominada de la dependencia) y posestructurales (influenciadas por los estudios culturales y los estudios de género, el análisis del discurso, desde donde emerge una perspectiva llamada posdesarrollo). Todo ello hace más compleja la gestión de acuerdos socialmente legitimados y duraderos en el tiempo sobre lo que implica coadyuvar a desarrollar una ciudad.

Otra de las pautas rastreadas a la hora de hablar de gestión urbana en los tiempos actuales tiene que ver con cierto estatuto epistemológico que configura la noción, esto es, su cualidad inter y transdisciplinaria ${ }^{6}$. Pasar de "objetos" de conocimiento producidos bajo dominios disciplinares a "objetos" de conocimiento construidos por lógicas más relacionales y complejas, sin duda, presenta un nuevo reto. El de comprender qué podría estar significando este cambio de perspectiva a la hora de pensar y actuar en las ciudades. Es común encontrar definiciones de gestión

\footnotetext{
${ }^{6}$ Lo disciplinar puede ser comprendido como la perspectiva del saber que tiene definido un objeto de conocimiento y un método compartido de indagación. Lo interdisciplinario, por su parte, alienta el diálogo desdela diversa generación de interpretaciones que sobre el fenómeno de estudio pueden producir las disciplinas científicas. Por último, lo transdisciplinario auspicia la construcción de objetos de investigación y métodos de indagación desde la confluencia heurística de las diferentes ciencias. Para profundizar sobre el tema véase Flórez-Malagón \& Benavides (2002), Nicolescu (1996) y Morin (1994).
}

urbana que invitan al trabajo armónico, que se deje de lado la pretensión de jerarquizar el conocimiento, y en lugar de ello se establezcan relaciones más horizontales entre los diferentes dominios del saber. Desde estos enfoques que buscan la inter y la transdisciplinariedad a la hora de conceptuar sobre la gestión de las ciudades, emergen postulados que aluden al desarrollo integral de los asentamientos humanos principales como propósito central de la gestión. La forma de ir operacionalizando tal posicionamiento tiene que ver con referir que existe un entrecruzamiento entre las dimensiones del desarrollo, a saber, entre lo social, lo económico, lo ambiental, lo cultural y lo político-institucional.

Es este sentido se afirma que existen "medios" que pueden auspiciar, o mejor dicho, pueden devolver los vínculos perdidos, en la medida en que el modelo de conocimiento moderno privilegió el paso de la búsqueda de la unidad de conocimiento, mathesis universalis, a la búsqueda de saberes más especializados, lo que se conoce como disciplinamiento del saber. "Medios" que están directamente relacionados con la gestión, por ejemplo, de lo público, de lo tecnológico. En el primer caso, su apuesta implica, entre otros aspectos, "tener una línea de investigación de políticas públicas, donde el problema de la gestión sea mucho más fuerte y no sólo lo sea la formulación de políticas públicas" [ex.: De acuerdo con Duarte (Firmino, 2013)]. Y en el segundo, por ejemplo, implica hacerse preguntas sobre cuáles son las transformaciones que inducen los avances de las nuevas tecnologías de la información y la comunicación - NTIC, en los territorios, en los espacios de la ciudad. Frente a esto el investigador Rodrigo Firmino considera lo siguiente: "cómo las nuevas tecnologías afectan la comprensión y el uso del espacio urbano contemporáneo. Cómo la gestión urbana maneja el espacio urbano, me parece que es de una importancia fundamental, comprender cómo se transforma, cómo se cambia el espacio" [ex.: De acuerdo con Firmino (2012)]. Desde esta perspectiva que concibe lo urbano como una red de relaciones, la gestión de la ciudad se erige como la posibilidad de articular niveles de la realidad plurales, que impliquen lo físico, lo virtual, lo simbólico, lo subjetivo. Niveles de realidad que no soslayen el carácter híbrido y mutante de aquello que podría implicar hoy en día lo espacial.

Hasta aquí se han planteado algunas pautas que los investigadores de la gestión urbana vienen 
privilegiando cuando se acercan a este "objeto" de conocimiento denominado gestión urbana. Sobresalen las pautas construidas a mitad del siglo anterior, inducidas por cierto "espíritu del tiempo", por cierta valoración social que auspicia la búsqueda permanente de modernización en los asentamientos humanos. Desde esta orientación a la gestión urbana la precedía un conocimiento racional, un saber experto. Las crisis desatadas por el desbordado crecimiento de las ciudades en las últimas décadas del siglo anterior, condujo, entre otros aspectos, a generar otras pautas de entendimiento sobre la gestión de lo urbano. Es así que se re-valorizan las apuestas de la administración tradicional y la creencia en la institución del mercado como regulador del bienestar social. Contemporáneamente, emergen pautas de gestión urbana que la entienden como una interfaz entre pensamiento y acción, como reivindicación de la capacidad de creación de los sujetos sociales, esto es, de la agencia cotidiana. Otras perspectivas la ligan con estatutos más epistemológicos como su carácter inter y transdisciplinario. Los defensores de tal propuesta de entendimiento dirían que la gestión de lo urbano va más allá de lo disciplinar. Es por ello que se asume como un dispositivo con el potencial de devolverle vínculos a la vida social, esto es, de devolverle vínculos perdidos a la ciudad.

\section{Estudios que aportan al análisis didáctico}

El recorrido que se ha venido planteando en términos de contenido, es decir, de aquello que se enseña, es uno de los componentes centrales en el proceso de enseñanza, al menos para quienes escriben este artículo. Enseñar Gestión Urbana, en este caso, indiscutiblemente nos lleva a debates teóricos, posturas políticas, visiones de vida. En este enseñar, desde una perspectiva didáctica y psicológica, se convoca a pensar su "arte" y preguntarse: ¿Qué es una buena enseñanza? ¿Es posible agenciar una enseñanza eficaz? ¿Cómo se puede entender la formación docente? Dichas cuestiones apenas son un pretexto para ahondar en algunas de las relaciones presentes entre la gestión de lo urbano y los procesos conducentes a su enseñanza: ¿Quiénes forman a los gestores urbanos? ¿Qué privilegian en sus métodos de enseñanza? ¿Un estilo docente influye en posturas asumidas por futuros gestores urbanos? Se pretende entablar un diálogo sobre algunas de las cuestiones de Gestión Urbana, planteadas ahora en clave de su enseñanza, para que de esta forma, quizá, se agencien "mejores" procesos de enseñanza de la Gestión Urbana.

Específicamente, las investigaciones revisadas aportan lo siguiente. Interpretando a Vera María Candau (1987) son las dimensiones técnica y humanística las que han prevalecido en el análisis de la enseñanza. La primera se entiende como una acción intencional y sistemática que busca provocar las mejores condiciones para que se propicie el aprendizaje a partir de unos buenos objetivos, selección de contenido, método de enseñanza y evaluación. La segunda tiene como centro del proceso la relación interpersonal en lo que corresponde a la adquisición de actitudes. Sin embargo, desde su punto de vista, dichas dimensiones son necesarias pero no suficientes, ya que es necesario considerar una nueva que se ha "silenciado": la política. Por lo tanto, las dimensiones claves, que muestran que un profesional es buen profesor, serían tres: la técnica (énfasis en saber), la humana (énfasis en ser) y la política (énfasis en la ética y las condiciones reales en que se propicia la enseñanza). Sin duda alguna, y tras indagar en las aulas de clases de varios posgrados, es evidente que las organizaciones educativas y el profesor privilegian las dimensiones técnica y humana. Pocas veces se observa en el profesor -por lo menos en lo que sucede en las aulas de clases- un posicionamiento político, una invitación al debate público en sus ejercicios de aula, esto es, una postura clara, argumentada y crítica frente a los vaivenes de nuestras sociedades. Efectivamente su posicionamiento es de un experto que relata una historia "leída y construida a través de los años", en la que no se conoce su participación, con una serie de autores y fechas, que parecieran ajenos a las vivencias del profesor, de la organización y del estudiante.

En relación a los aspectos que constituyen a un profesor experto, Gudmundsdóttir \& Shulman (2005) identifican cuatro características centrales: conocimiento experto de su disciplina; las oportunidades de redefinir su asignatura a partir del número de veces que la ha ofrecido; la segmentación y estructuración del currículum le permite conocer pros y contras de cada enfoque; y percibir las unidades como un todo y no por partes, lo cual, resaltan los autores, puede ser una magnífica técnica de contar relatos. De otra parte, Allen \& Ryan (1978), y Oliva \& Henson (1989) otorgan valor al profesor 
que reconoce el comportamiento atento y que da especial atención al estudiante en estos aspectos: interés, paciencia y sentido de la equidad. Los autores registran estas características fundamentales: conocer y estar preparado en su materia; tener amplia educación general, sentido del humor y mentalidad abierta; tener aspecto pulcro; conocer los principios básicos del proceso de aprendizaje; tener capacidad de inducción, conclusión, silencio, recursos no verbales, de forzar la participación, de plantear preguntas con fluidez, ilustrar y utilizar ejemplos, repetir de manera planificada, terminar las explicaciones, entre otras. Veamos ahora como algunos de estos aspectos descritos sobre el profesor experto se concretan en la experiencia de vida y en el testimonio de Clovis Ultramari, profesor brasileño:

[...] yo insisto con los estudiantes que debemos entender todos los escenarios sociales, políticos e históricos, pero lo que verdaderamente nos hace urbanistas es la transformación de toda esa complejidad social de una manera física. No es un concepto esto, es una idea que yo tengo y que creo importante, esa es mi dirección: esta relación con la cuestión física, construida, concreta de la ciudad. Porque yo no soy Sociólogo no tengo formación en Sociología, yo soy Arquitecto [...] (Firmino, 2013).

Stella Cols, desde la didáctica, considera que un buen profesor es aquel que "interpreta la marcha de los acontecimientos, juzga los elementos de juego, y toma decisiones que pueden incluir la revisión de su método" (Cols, 2004, p. 6). Para Fenstermacher (1986) la tarea de un profesor es hacer "estudiantar", es decir, generar en el estudiante el deseo por el estudio y por mejorar su capacidad de hacerlo. En el mismo sentido se manifiesta Brophy (1998, apud Hernández \& Sancho, 1993) para quien lo importante es que el profesor explique a sus estudiantes el por qué es interesante el tema o idea que presenta. De igual forma logre estimular la curiosidad mediante paradojas, contradicciones, incongruencias y, que a partir de la reflexión, les ayude a adquirir mayor conciencia sobre sus procesos y dificultades. Desde el campo de la evaluación Stake (2011) considera fundamental la capacidad crítica del profesor, capacidad que considera se ha ido perdiendo. El siguiente relato de un estudiante ilustra lo que significa para la experiencia de formación de un estudiante el ejercicio de un docente experto, el cual logra estimular la pasión por el conocer, esto es, logra "despertar" el deseo por estudiar un tema en particular, por curiosearlo, por vivir intensamente el proceso de compresión del fenómeno que interesa, en este caso la ciudad.

[...] por su calidad me parece que es una materia con la que se debe iniciar una maestría en Gestión Urbana. Además la calidad del docente, de persona que es, entonces te lleva a soñar, a soñar en la gestión, a soñar e idealizar una gestión urbana a nivel de lo complejo que es la ciudad pero viendo lo maravilloso que es el componente social. Empieza a hablar de que todos conformamos ciudad, de que todos somos ciudad, y que hacemos parte de la ciudad. La ciudad no es de un solo individuo, no es del Alcalde, no es de los políticos, es de todas las personas que vivimos en ella [...] (Estudiante 1, Firmino, 2013).

Teresa de J. Cañedo et al. (2008) a partir de un estudio exploratorio y fenomenológico refieren una serie de aspectos que debe tener en cuenta el profesor de posgrado para que su materia sea aprendida. Se destaca la necesidad de realizar actividades que confronten lo que los estudiantes realizan en sus actividades laborales. De igual forma los autores sugieren la creación de momentos de trabajo grupal que aporten al desarrollo profesional y personal, así como la vinculación de metodologías en las que se involucre el manejo de tecnologías. Por último se llama la atención en la necesidad de propiciar la coevaluación, "entendida como la evaluación que se realiza entre los estudiantes respecto a la forma de trabajo en las distintas actividades" (Cañedo et al., 2008, p. 70). En este sentido la experiencia de aula del profesor argentino Juan Carlos Pérgolis ejemplifica el uso de la tecnología de la imagen como dispositivo que interpela a los estudiantes en su quehacer no sólo formativo sino también profesional y personal.

Si el propósito es sensibilizarlos con la ciudad, el método es totalmente arbitrario. Mira, yo llevo dictando clases en Colombia desde el año 78 [...] el método es totalmente aleatorio. Por eso lo primero que yo miro como urbanista es la literatura, miro más la literatura que el urbanismo porque la literatura tiene métodos por una parte rápidos, por otra 
parte, capaces de generar imágenes fuertes de la ciudad. Por otro lado genera estructuras inestables que se pueden cambiar con facilidad. El urbanismo se acostumbró a las grandes infraestructuras inamovibles, sobre todo el urbanismo moderno. Eso es el inicio de la organización de la clase. Luego, ¿a quién va dirigida? en el caso nuestro la mayoría son Arquitectos. Gente que se forma en la cultura de la imagen, es decir, algo lo entienden si leen imágenes. Gente que por su formación no tienen esas imágenes previas para tener las representaciones (Abogados, Sociólogos y demás). La reflexión sobre la ciudad implica imágenes [...] hay que presentarles imágenes como referencia, en este caso de Bogotá, frente a otras ciudades (la plaza de Bolívar en Bogotá o la plaza Moreno en La Plata). Eso es la segunda parte; abrir el pensamiento no solo a una ciudad sino a rasgos que es la expresión de la comunidad en el territorio [...] luego viene la narrativa de la clase [...] (Firmino, 2013).

Cashin (1995) alerta sobre las características que han de ser controladas a la hora de evaluar al profesor. Organiza estas variables en tres aspectos: el del profesor (edad, experiencia docente, género y personalidad, estatus dentro de la facultad y producción investigativa), el del estudiante (edad, género, nivel y personalidad), y otros (tamaño del grupo, hora, época del semestre, entusiasmo del profesor, calidad de trabajo, dificultad del curso). Por su parte Carreño (2012), después de revisar fuentes secundarias y observar clases de manera etnográfica, concluye que para el caso de los posgrados sobre desarrollo (en los que se subsume la gestión urbana) se requieren habilidades y prácticas que alienten la relación teoría-práctica, capacidad para la reflexión-acción, y abordajes que reconozcan la heterogeneidad, la multidisciplinariedad, lo multiregional y lo multicultural. En este sentido, para que los estudiantes aprendan, han de tener experiencia en el tema y, dentro de las actividades de enseñanza, contar con salidas de campo. Los profesores deben conocer el contenido, han de tener claro los desequilibrios que generan las prácticas de la enseñanza y reconocer que el oficio de enseñar se recrea en la práctica. El testimonio de Fabio Duarte, profesor brasilero, da pistas sobre este aspecto en particular:

[...] pero normalmente ya tenemos un programa, un marco teórico. Nos encanta imaginar que la ciudad es un artefacto tecnológico, y cuando decimos la ciudad no solamente nos referimos a la parte física sino a la forma en que la gente vive, trabaja, se relaciona. Todo eso hace parte de un gran artefacto tecnológico que por supuesto está directamente vinculado a una dimensión económica y social de escala global. Entonces la ciudad es un artefacto tecnológico y tiene intenciones claras de lo que debe ser [...] (Firmino, 2013).

Para Carreño se hace urgente pensar la enseñanza en posgrados reconociendo que lo que acontece en el salón de clases impacta en la formación profesional y personal del estudiante. De ahí que sea necesario programar y llevar a la acción una enseñanza con dirección pero con oportunidad de elegir entre variadas actividades. Estas actividades plurales permiten introducir ritmo dinámico en el proceso de enseñanza. Se hace necesario revisar el método de enseñanza para que involucre un plan de estudios basado en la reflexión-acción (como espacio para preocuparse sobre lo que se conoce y sobre lo que otros conocen, así como escenario para aprender haciendo). La experiencia del profesor brasileño Rodrigo Firmino con la asignatura "Ciudad y Tecnología” ilustra la necesidad de programar y realizar una enseñanza desde la innovación permanente en el aula.

[...] una clase nunca es igual a otra, entonces pienso en todo lo que pierden los que no están al inicio de la clase, pueden perder cosas que nunca más voy a hablar; entonces espero un poco, arreglo el computador. Siempre hablo un poco sobre lo que ha pasado en el curso hasta ese momento y cómo se va a desarrollar en la siguiente clase, y empiezo la exposición. Como estas clases son muy largas, no se puede hacer una exposición por toda la tarde, no, porque no tienen como concentrarse por más de dos horas, entonces les propongo otros trabajos: videos, periódicos, rueda de discusiones. Me gusta mucho variar (Firmino, 2013). 
La etnografía realizada en una de las clases en el nivel de posgrado permite observar los cambios de ritmo en la enseñanza, aspecto crucial para ir involucrando activamente a los estudiantes en el proceso de formación:

Al terminar la presentación comienza a rodar una película, son las 8:42 a.m., todos están en silencio. Acto seguido todos cambian de posición, ya no miran hacia la pared sino hacia el tablero. El tercer profesor habla mientras mira a los estudiantes, se siente ruido afuera. El profesor utiliza las manos en su expresión, mira la mesa y habla pensando en torno a lo visto en la película. Son las 9:01 a.m. habla sobre el uso, mientras tanto los estudiantes toman nota, algunos a mano y otros en computador. El profe se retira el saco, sigue hablando, se pasa la mano por su cabeza. Todos siguen con atención al profesor [...]

\section{Aportes a la enseñanza sobre gestión urbana}

En la búsqueda por articular lo que se enseña por Gestión Urbana y la manera con la que se privilegia su enseñanza, entre 2011 y 2013 se realizó un rastreo bibliográfico que permitió identificar algunos artículos significativos sobre la cuestión de la enseñanza de la gestión urbana. Frey (2012) reflexiona sobre la enseñanza de la gestión urbana en la asignatura "Gobierno de las instituciones locales". Califica la gestión urbana como un desafío colectivo, razón por la cual releva la formación que el gestor tenga en términos del "arte de la negociación y articulación entre la política y la administración pública, de intermediar y dialogar con los diferentes saberes y sectores, de comunicarse e interactuar con los actores de la sociedad civil, el empresariado y los ciudadanos" (Frey, 2012, p. 34). Toda esta gama de habilidades que debe fortalecer el futuro gestor urbano se conjuga con "sensibilidad y compromiso con las exigencias de la democracia, la justicia social y la sostenibilidad de la sociedad". (Frey, 2012, p. 34) ¿Cómo generar o potenciar en el estudiante sea o no tangible- dicha sensibilidad y compromiso como principios claves para la sociedad y su avance académico y práctico?
Uno de los grandes dilemas y retos para la enseñanza de este tema, agrega Klaus, es quela realidad social investigada no necesariamente "corresponde a la realidad imaginada por los conceptos teóricos, sobre todo cuando son importados de otros contextos y tradiciones científicas, muchas veces originarios de países en desarrollo" (p. 39). Es por esta razón que el proceso de enseñanza en posgrados es co-formativo. Es un proceso entre profesionales que se congregan en un espacio y ponen en interlocución su Saber (S), su Experiencia (E) y un acumulado de Conocimientos (C). En el caso del profesor, este acumulado ha sido sistematizado durante toda su trayectoria docente, ha tenido recortes, se ha vinculado con sus experiencias y se ha llegado a la construcción de unos contenidos $(\mathrm{S}+\mathrm{E}+\mathrm{C}=$ Contenido) que son los que comparte y debate con sus estudiantes.

A las categorías de negociación, comunicación y diálogo que propone Frey para la enseñanza de la gestión urbana, Claudia Inés Carreño adiciona en su artículo "La gestión urbana. Una aproximación a la noción desde su enseñanza", una preocupación por la reflexión-acción. Afirma que la noción debería enseñarse "como un proceso de reflexiónacción que busque la construcción de la vida social, privilegiando la mejora de la vida de las personas y de sus entornos bajo parámetros democráticos, justos y duraderos" (Frey, 2012, p. 10). Desde una mirada didáctica, propone que la gestión urbana también se piense en términos de método e incorpore tres elementos centrales para animar en el salón de clases: experiencias, negociación y relaciones. Y finalmente se pregunta: ¿Es posible empoderar a los estudiantes, en términos de participación, democracia y derechos, con una propuesta metodológica más activa?

Jorge Inzulza (2011) en "Recuperando el derecho al espacio público desde la enseñanza de la arquitectura y el diseño urbano" presenta una reflexión aplicada y una reivindicación del diseño urbano a partir de la realización de un Taller de Diseño Urbano. El ejercicio lo realizó a partir del análisis del río Mapocho, ubicado en la ciudad de Santiago (Chile), como elemento que permite analizar una propuesta integral de diseño urbano. Como resultado del ejercicio académico, que se propone en el artículo, se encuentran tres aspectos centrales: a) se destaca el papel de la Arquitectura y el Diseño Urbano que, enseñadas como disciplinas, facilitan el entendimiento de la ciudad; b) el ejercicio académico (de reflexión, debate y comprensión) permite 
poner en discusión la importancia de los "espacios públicos como derecho propio de cada ciudadano, y su aplicación a través del diseño urbano como la disciplina que permite dar «forma y sentido» al hecho urbano" (Inzulza, 2011, p. 38); y, c) el derecho a la ciudad va más allá del acceso a lo existente; es el derecho a modificar y a transformar la ciudad como ejercicio colectivo.

En la búsqueda de diseñar un enfoque para la enseñanza del urbanismo, Moya González (1997), en su ensayo "La enseñanza del urbanismo" propone dos preguntas básicas: ¿qué enseñar? y ¿cómo enseñamos? En términos del "qué", Moya González (1997) considera que la enseñanza del urbanismo para arquitectos ha de atender la base formativa de los estudiantes y no requiere de una carrera específica debido a la complejidad de la cuestión urbana y territorial. Específicamente debe abordar una formación que "coordine y aglutine conocimientos y que a la vez les brinde capacidad de síntesis" (Moya González, 1997, p. 35). En términos del "cómo" hace referencia al Taller "como método de trabajo proyectual que sin perder la carga teórica habitual se plasme en ejercicios propositivos de ordenación espacial" (Moya González, 1997, p. 138). Específicamente para el posgrado agrega que para complementar la formación ha de ser interdisciplinario, y debe contrastar enfoques y formas de trabajo en equipo. Y es por ello que se pregunta: ¿Requiere el posgrado, especialmente el centrado en la gestión urbana, especificidad de la disciplina, formas interdisciplinarias de trabajo o miradas mucho más multidisciplinarias que pongan en terreno real el debate?

¿Es posible empoderar a los estudiantes, en términos de participación, democracia y derechos, con una propuesta metodológica más activa? ¿Cómo comprender ese tránsito entre el saber sabio, el saber enseñado y lo que efectivamente aprende un estudiante? ¿Cómo identificar si realmente se ha alcanzado una co-formación con sensibilidad social y capacidad de reflexión crítica? ¿Cuál es el papel del profesor en este proceso de "dar sentido" a lo que se enseña? Son algunas de las preguntas que emergen al poner en diálogo las nociones de gestión urbana y de enseñanza.

\section{Algunas ideas y preguntas, sin pretexto de concluir}

En la primera parte del artículo se destacaron algunas pautas contemporáneas que han contribuido a otorgar sentido a la noción de la gestión urbana, a saber, la búsqueda de la modernización de los asentamientos humanos de la mano del conocimiento experto, la administración de los procesos urbanos y el privilegio del mercado como institución reguladora de la vida social. Recientemente otras pautas han emergido, entre las que destacan, el énfasis en la capacidad creadora de los sujetos y las posibilidades de las estrategias de producción de conocimientos más allá de las disciplinas, esto es, las prácticas inter y transdisciplinarias. Estas últimas pautas sin duda han inducido procesos de enseñanza de la gestión urbana más significativos y, a la vez, introducen desafíos a dicha práctica. En términos generales las reflexiones didácticas, descritas en el apartado dos, mostraron que una "buena enseñanza" necesita que el profesor agencie habilidades personales, criterios técnicos, virtudes éticas y cualidades políticas. En lo personal hace referencia -entre otros- a la edad, nivel cultural, personalidad, presentación personal. En lo técnico se destaca el conocimiento sobre el tema, la preparación en su materia, la capacidad para inducir, preguntar, ejemplificar, hacer cierres de temas, la forma de enseñar. En lo ético se alude a la relación interpersonal que se genera y posibilita adquirir actitudes o generar cambios de comportamiento. Y en lo político existe una preocupación por las condiciones reales en que se dan las prácticas de la enseñanza.

La enseñanza de la gestión urbana implica algunos desafíos relativos al mundo actual. El profesor debe dejar de pensar el aula de clase como en un sistema educativo en sí mismo, para comenzar a generar procesos tendientes a que el aula de clase se perciba como un nodo de una red educativa que va desde la ciudad a un mundo más global. En este sentido, los procesos de gestión urbana descritos anteriormente aportan mucho de manera significativa, dado que se constituyen como "medios" para interconectar niveles de la vida social que, desde otras perspectivas, se consideraban carentes de vínculos, carentes de relaciones.

Por último, surgen algunas preguntas sobre la enseñanza en gestión urbana: ¿Requiere el 
posgrado, y en especial el referido a la gestión urbana, enseñanzas más ligadas a la especificidad de las disciplinas, a formas más interdisciplinarias de trabajo, a miradas mucho más multidisciplinarias que pongan en terreno real el debate teórico? ¿Es posible empoderar a los estudiantes, en términos de participación, democracia y derechos, con propuestas de enseñanza más activas? ¿Cómo generar o potenciar en el estudiante el dominio del "arte" de la pregunta, la capacidad de interrogarse, de pensar la ciudad más allá de la percepción inmediata? ¿En este sentido cual puede ser el rol del profesor en su proceso de enseñanza? ¿Cómo comprender el tránsito entre el saber sabio, el saber enseñado y lo que efectivamente aprende un estudiante? ¿Qué tipo de dispositivos pedagógicos pueden contribuir a evaluar el grado alcanzado de la co-formación en el salón de clase? ¿Existirán relaciones entre los procesos de enseñanza escogidos por el docente y los grados de sensibilidad social y capacidad de reflexión crítica alcanzada por los estudiantes? ¿Cuál podría ser el papel del profesor en este proceso de "dar sentido" a lo que se enseña? ¿Hasta qué punto la enseñanza de la gestión urbana está configurando espacios de aula realmente significativos?

\section{Referencias}

Allen, D., \& Ryan, K. (1978). Microenseñanza - Una nueva técnica para la formación y el perfeccionamiento docentes. Buenos Aires: Editorial El Ateneo.

Balbo, M. (2003). La nueva gestión urbana. In R. Jordan, \& D. Simioni (Orgs.), Gestión urbana para el desarrollo sostenible en América Latina y el Caribe (p. 133-139). Santiago de Chile: Naciones Unidas-CEPAL; Cooperazione Italiana.

Camargo, A. (2012). Anotaciones sobre la historia de la Maestría en Gestión Urbana. Papeles de Coyuntura, 33, 3-6.

Camou, A. (2009). La universidad argentina en discusión. In S. Gvirtz, \& A. Camou (Orgs.), La universidad argentina en discusión. Sistemas de ingreso, financiamiento, evaluación de la calidad y relación Universidad-Estado (p. 21-44). Buenos Aires: Granica.

Candau, V. M. (1987). La didáctica y la formación de educadores - de la exaltación a la negociación. In V. M. Candau (Org.), La didáctica en cuestión (p. 14-23). Madrid: Notigrad S.A.
Cañedo, T. J., Rubalcava, A. E. F., Calderón, D. V., \& Penaflor, C. Z. (2008). Evaluando la enseñanza en el posgrado. Reencuentro, 53, 63-74.

Carreño, C. I. (2012). Lo pedagógico en los posgrados sobre desarrollo. Dos estudios de caso (Tesis doctoral). Universidad Nacional de La Plata, La Plata.

Carreño, C. I. (2013). Prólogo. In: C. I. Carreño (Ed.), Gestión Urbana en América Latina. Debates desde la reflexión-acción (p. 11-47). Bogotá: Universidad Piloto de Colombia.

Cashin, W. E. (1995). Student ratings of teaching: the research revisited (Idea Paper no. 32). Manhattan: Kansas State University; Center for Faculty Evaluation and Development.

Cataño, G. (2008). Estudios de posgrado en educación. Colombia: Biblioteca virtual del Banco de la República. Recuperado en día de enero de 2008, de http://www. lablaa.org/blaavirtual/bibliotecologia/bibliotecas/estud. htm.

Cols, E. (2004). Programación de la enseñanza, ficha de cátedra Didáctica I. Buenos Aires: Universidad de Buenos Aires.

Domínguez, M. A. (2011). Los modos de intercambios de significados en clases de física secundaria - Procesos de negociación (Tesis de doctorado). Universidad Nacional de La Plata, La Plata.

Durán, D. A. (2012). Gestión y desarrollo social: reflexiones desde la comunicación-educación. Manuscrito presentado para su publicación.

Fayad, R. (2010). Anotaciones para una reflexión sobre la educación superior en Colombia. Revista Med, 18(1), 123-133.

Fenstermacher, G. D. (1986). Tres aspectos de la filosofía de la investigación sobre la enseñanza. In: M. C. Wittrock (Org.), La investigación de la enseñanza I: Enfoques, teoría y métodos (p. 150-176). Barcelona: Paidós Educador.

Firmino, R. (2012). Entrevista ao autor do artigo. Bogotá. Manuscrito inédito.

Firmino, R. (2013). Entrevista ao autor do artigo. Curitiba. Manuscrito inédito.

Flórez-Malagón, A. G., \& Benavides, C. M. (Eds.) (2002). Desafíos de la transdisciplinariedad. Bogotá: Pontificia Universidad Javeriana; Instituto Pensar. 
Frey, K. (2012). Enseñanza e investigación sobre gestión urbana: algunas anotaciones conceptuales y el relato de una investigación con alumnos de la Maestría en Gestión Urbana. Papeles de Coyuntura, 33, 32-39.

García Cruz, O. M., \& Cárdenas, L. A. C. (2004). Espacio público: el reto de la gestión. Revista Bitácora Urbano Regional, 8(1), 103-109.

García de Fanelli, A. M., Serna, R. K., Mendiola, G. A., Gardía, R. R., \& Trombetta, A. M. (2001). Entre la academia y el mercado: posgrados en ciencias sociales y políticas públicas en Argentina y México. México: Colección Biblioteca de la Educación Superior; ANUIES.

GEUR (2012). Guía básica de Gestión Urbana. Recuperado en día de junio de 2013, de http://www.geur.com.mx/ geur/guiabasica.pdf.

Guadilla, G. C. (2003). Balance de la década de los '90 y reflexiones sobre las nuevas fuerzas de cambio en la educación superior. In: M. Mollis (Org.), Las universidades en América Latina: ¿reformadas o alteradas? (p. 13-37). Buenos Aires: Clacso.

Guadilla, G. C. (2014). Financiamiento de la educación superior en América Latina en educación superior en el mundo 2006 - financiamiento de las universidades. Madrid: Ediciones Mundi-Prensa.

Gudmundsdóttir, S., \& Shulman, L. S. (2005). Conocimiento didáctica en Ciencias Sociales. Revista de Currículum y Formación de Profesorado, 9(2), 1-12.

Hernández, F., \& Sancho, J. (1993). Visiones sobre el aprendizaje y la enseñanza: La búsqueda de un dorado eficaz. In: F. Hernández (Org.), Para enseñar no basta con saber la asignatura (p. 19-32). Barcelona: Editorial Paidós.

Inzulza, J. (2011). Recuperando el derecho al espacio público desde la enseñanza de la arquitectura y el diseño urbano. Revista de Arquitectura - Espacio Público y Ciudadanías, 24, 35-40.

Jordan, R., \& Simione, D. (1998). La planificación y la géstión urbana local. In Cepal (Org.), Ciudades intermedias de América Latina y El Caribe: propuesta para la Gestión Urbana (p. 120-128). Santiago de Chile: Cepal.

Kennedy, M. (2008). Teachers Thinking about practice. In: T. Good (Org.), 21 Century Education: a Reference Handbook (Vol. 2, p. 21-28). London: Sage Publ.
Krotsch, P. (2001). Educación superior y reformas comparadas. Buenos Aires: Universidad Nacional de Quilmes Editorial.

Marquis, C., Spagnolo, F., \& Valenti, G. (1998). Desarrollo y acreditación de los postgrados en Argentina, Brasil y México: Textos para una mirada comparativa. Buenos Aires: Ministerio de Cultura y Educación.

Matallana, M. A., \& Varela, S. P. (2006). Tendencias de los estudios de posgrado en Colombia. Bogotá: Cendex; Pontificia Universidad Javeriana.

Messina, G. (1999). Investigación en o investigación acerca de la formación docente: un estado del arte en los noventa. Revista Iberoamericana de Educación, 19, 145-207.

Morin, E. (1994). Carta de la transdisciplinariedad. Portugal: Convento de Arrábida.

Moya González, L. (1997). La enseñanza del urbanismo. Urban, 1, 135-139.

Nicolescu, B. (1996). La transdisciplinariedad. Manifiesto. México: Multiversidad Mundo Real Edgar Morin A. C.

Oliva, F., \& Henson, K. T. (1989). ¿Cuáles son las competencias genéricas esenciales de la enseñanza? In J. Gimeno Cristán, \& A. Pérez Gómez (Orgs.), La enseñanza: su teoría y su práctica (p. 357-363). Madrid: Akal.

Ortega, Á. C. (2008). Algunas consideraciones sobre la definición, importancia y complejidad de la gestión urbana: a manera de introducción. In Grupo de Investigaciones, $L a$ gestión urbana: debates y aplicaciones (p. 11-23). Bogotá: Universidad Piloto de Colombia.

Páez, V. A. (2013). Condiciones para una gestión pública compartida. Documento borrador de Trabajo de Grado Cider. Bogotá: Universidad de los Andes.

Parraguez, L., Loza, G. R., \& Bellei, M. S. (2006). ¿Cómo se piensa la ciudad? Análisis crítico de un siglo de gestión y planificación urbana. Revista Eure, XXXII(96), 135-140.

Stake, R. E. (2011). ¿Los profesores del futuro devolverán el poder crítico a las universidades? In Conferencia en el marco del III Coloquio Internacional de RIIED. Bogotá: Centro de Investigación y Formación en Educación (Cife) de Uniandes.

Recibido: Ene. 23, 2014

Aprobado: Mayo 8, 2014 